\title{
On the Expressivity of Optimality Theory versus Ordered Rewrite Rules
}

\author{
Brian Buccola \\ Department of Linguistics \\ McGill University \\ brian.buccola@mail.mcgill.ca
}

\begin{abstract}
I prove that there are phonological patterns which are expressible by ordered rewrite rules but not by any Optimality Theoretic (OT) grammar whose constraint set contains only markedness constraints and single-state faithfulness constraints, i.e. faithfulness constraints that assign violation marks to pairs of single input-output segments in correspondence, with no reference to other segments in the input or output. The intention is to capture formally the widespread intuition that certain opaque patterns, which are expressible by ordered rewrite rules, are problematic for classic, or traditional, OT.
\end{abstract}

\section{Introduction}

Generative phonology takes the phonetic form of an utterance to be the surface realization (or output) of an abstract, underlying phonological form (or input). Natural languages therefore exhibit input-output patterns, which the phonologist is tasked with describing in a formal, algorithmic way. Phonologists have largely described these patterns using two types of grammar: (i) serial rule grammars, e.g. as laid out in The Sound Patterns of English (SPE) [1], in which an input is mapped serially to intermediate outputs and finally to a terminal output via ordered, context-sensitive rewrite rules; and (ii) parallel constraint grammars, e.g. as in Optimality Theory (OT) [2], in which an input is mapped directly to that output which is optimal with respect to a set of ranked constraints, with no intermediate mappings (i.e. everything happens in parallel).

This paper addresses the following two questions: (i) Are there input-output patterns that can be expressed by one type of grammar but not by the other, and (ii) if so, are those patterns attested in natural language? The first is a formal question about the classes of input-output relations expressible by the two formalisms. The second is an empirical question that bears on whether phonologists should favor one type of grammar over another on the basis of empirical coverage.

Under the assumption that a rule may not rewrite within that part of a string which it has already rewritten, ${ }^{1}$ the patterns expressible by ordered rewrite rules

\footnotetext{
${ }^{1}$ Nowhere in this paper is this assumption violated. Henceforth, when I write "ordered rewrite rules," I mean rule-based grammars in which this assumption is in place.
} 
correspond exactly to the regular relations $[3,4,5] .{ }^{2}$ Without any restrictions, the patterns expressible by OT grammars include non-regular relations, i.e. patterns that are inexpressible by ordered rewrite rules $[6,7] .^{3}$

What is not known is whether every regular relation is expressible by some OT grammar [10]. Put differently, the question is still open whether every pattern expressible by ordered rewrite rules is expressible by some OT grammar.

Nevertheless, there is a widespread intuition that certain opaque phonological patterns, which are expressible by ordered rewrite rules (hence, they are regular), are problematic for classic [11], or traditional [10], OT grammars, consisting of just two basic types of constraints (output markedness and input-output faithfulness). However, to my knowledge, no one has yet formally proved this claim. Such a proof could reach two possible conclusions: (i) there are regular relations that are completely inexpressible by classic OT grammars, or (ii) all regular relations are expressible in principle by classic OT, albeit sometimes only with ad hoc, linguistically unmotivated, but formally sound constraints.

In this paper I define classic OT grammars as OT grammars that contain only markedness and single-state faithfulness constraints, i.e. faithfulness constraints that assign violation marks to pairs of single input-output segments in correspondence, with no reference to other segments in the input or output. These constraints include many, if not most, of the standard constraints proposed in the OT phonology literature, e.g. IDENT, MAX, and DEP. I then prove that there are regular relations that cannot be expressed by any such OT grammar.

Section 2 reviews SPE-style and OT-style grammars, as well as the notion of opacity. In section 3, I prove, using data from Canadian English, that there are input-output patterns which are expressible by ordered rewrite rules but not by any classic OT grammar, as defined here. In section 4, I discuss several other cases of opacity that are likewise provably expressible by ordered rewrite rules but not by classic OT grammars; and I discuss cases of opacity that seem, in principle, to be expressible by classic OT grammars. Section 5 concludes.

\section{Preliminaries}

In this section I lay out the architectures of SPE-style, rule-based phonologies and OT-style, constraint-based phonologies, and I explain the notion of phonological opacity. ${ }^{4}$

${ }^{2}$ The definition of a regular relation is not important for this paper; for details, see [4].

${ }^{3}$ Under certain assumptions, however, the patterns expressible by OT grammars lie within the regular relations $[8,9,10]$. These assumptions are (i) that both the constraints and the function GEN mapping inputs to sets of output candidates are regular, and (ii) that there is an upper bound on the number of violation marks assigned by any constraint. Without either assumption, OT can describe non-regular relations. I thank an anonymous referee for helping me to clarify these points.

${ }^{4}$ Many phonologists take features, rather than segments, to be phonological primitives. That is, rules and constraints are assumed to target natural classes of segments, rather than individual segments or arbitrary sets of segments. In principle, this means 


\subsection{Rule-based Phonology}

In an SPE-style, rule-based grammar, inputs are mapped to outputs via ordered, context-sensitive rewrite rules of the form

$$
A \rightarrow B / C-D
$$

read as, " $A$ is rewritten as $B$ whenever $A$ occurs immediately after $C$ and immediately before $D . "{ }^{5} A$ is the focus of the rule, while $C-D$ is the environment. The focus and environment together are called the input description. Under a single such rule, an input like $/ C A D /$ is mapped to the output $[C B D] .{ }^{6}$ If a rule does not effect any change from input to output, then it applies vacuously.

Since rules simply map strings to strings, it is possible for ordered rules to interact in the following ways (adapted from [12]). ${ }^{7}$

Definition 1. $\mathcal{R}_{1}$ feeds $\mathcal{R}_{2}$ iff $\mathcal{R}_{1}$ creates part of $\mathcal{R}_{2}$ 's input description.

Definition 2. $\mathcal{R}_{1}$ bleeds $\mathcal{R}_{2}$ iff $\mathcal{R}_{1}$ removes part of $\mathcal{R}_{2}$ 's input description.

Consider, for example, the rule

$$
\mathcal{R}: \text { aI } \rightarrow \Lambda \mathrm{I} / \text { t }
$$

where the vowel /aI/ before /t/ raises to $[\Lambda \mathrm{I}]$, and the rule

$$
\mathcal{F}: \mathrm{t}, \mathrm{d} \rightarrow \mathrm{r} / \mathrm{V}-\mathrm{V}
$$

where /t/ or /d/ between two vowels surfaces as a flap, [r]. If $\mathcal{F}$ is ordered before $\mathcal{R}$, then an input like / raitər/ is mapped by $\mathcal{F}$ to raIrər, which $\mathcal{R}$ maps vacuously to [rairər]. $\mathcal{F}$ bleeds $\mathcal{R}$ because if $\mathcal{F}$ were absent, then $\mathcal{R}$ would map / raitər/ to [rsitər]. The application of $\mathcal{F}$ before $\mathcal{R}$ removes part of $\mathcal{R}$ 's input description (_t), so that $\mathcal{R}$ no longer applies non-vacuously.

Feeding and bleeding can both be further subcategorized (adapted from [13]).

that feature-based phonologies are expressively more restrictive than segment-based ones. However, in this paper the distinction is immaterial: the patterns considered here are expressible by feature-based rules (hence, also by segment-based rules), and they are not expressible by segment-based constraints (hence, neither by featurebased constraints). Accordingly, I assume that rules and constraints generally target features, but in the proof in section 3, I allow constraints to target arbitrary (sets of) segments, demonstrating that even with such added power, classic OT grammars still cannot express the relevant patterns.

5 The more familiar notation in formal language theory is: $C A D \rightarrow C B D$.

${ }^{6}$ By convention, symbols between forward slashes are inputs (underlying forms), and those between square brackets are terminal outputs (surface forms). I write nonterminal, i.e. intermediate, outputs with no brackets at all. These conventions also apply to OT inputs and output candidates.

7 Rather than creating (removing) part of $\mathcal{R}_{2}$ 's input description, Baković [12] writes that $\mathcal{R}_{1}$ "creates (removes) additional (potential) inputs" to $\mathcal{R}_{2}$. The two formulations are equivalent. I prefer the former because it facilitates defining feeding and bleeding on environment and focus (below). 
Definition 3. $\mathcal{R}_{1}$ feeds on $\mathcal{R}_{2}$ 's focus iff $\mathcal{R}_{1}$ feeds $\mathcal{R}_{2}$ by creating $\mathcal{R}_{2}$ 's focus. $\mathcal{R}_{1}$ feeds on $\mathcal{R}_{2}$ 's environment iff $\mathcal{R}_{1}$ feeds $\mathcal{R}_{2}$ by creating part of $\mathcal{R}_{2}$ 's environment.

Definition 4. $\mathcal{R}_{1}$ bleeds on $\mathcal{R}_{2}$ 's focus iff $\mathcal{R}_{1}$ bleeds $\mathcal{R}_{2}$ by removing $\mathcal{R}_{2}$ 's focus. $\mathcal{R}_{1}$ bleeds on $\mathcal{R}_{2}$ 's environment iff $\mathcal{R}_{1}$ bleeds $\mathcal{R}_{2}$ by removing part of $\mathcal{R}_{2}$ 's environment.

The example above, with $\mathcal{F}$ ordered before $\mathcal{R}$, is one of bleeding on environment: the change effected by $\mathcal{F}$, i.e. mapping the /t/ in /rartər/ to [r], removes part of $\mathcal{R}$ 's environment ( $-\mathrm{t}$ ).

By convention, when I write that $\mathcal{R}_{1}$ "feeds (bleeds)" $\mathcal{R}_{2}$, it is implied that $\mathcal{R}_{1}$ is ordered before $\mathcal{R}_{2}$, even though strictly speaking, feeding (bleeding), as defined here, is independent of rule ordering. When a feeding (bleeding) rule is ordered after the rule it feeds (bleeds), the following terms are used.

Definition 5. $\mathcal{R}_{1}$ counterfeeds (counterbleeds) $\mathcal{R}_{2}$ iff $\mathcal{R}_{1}$ both feeds (bleeds) and is ordered after $\mathcal{R}_{2}$.

In the example above, if is ordered $\mathcal{F}$ after $\mathcal{R}$, then $\mathcal{F}$ counterbleeds on $\mathcal{R}$ 's environment.

These notions of rule interaction have been useful in characterizing phonological opacity. Kiparsky $[14,15]$ was the first to identify the phenomenon of opacity, defining it as follows, where "process" can be construed as a rewrite rule.

Definition 6. A process $\mathcal{P}$ of the form $A \rightarrow B / C-D$ is opaque to the extent that there are surface representations (outputs) of the form (i) $A$ in the environment $C \_D$, or (ii) $B$ derived by $\mathcal{P}$ in environments other than $C \_D$.

The idea is that opaque phonological generalizations are (i) generalizations that appear not to hold true of a surface form, or (ii) generalizations that are true of a surface form, but the motivation for their application is obscured.

These two types of opacity are typically associated with counterfeeding and counterbleeding rule ordering, respectively $[16,11,12]$. In counterfeeding, a later rule creates part of the input description of an earlier rule, such that the earlier rule seems not to have applied to the surface form, even though it matches the rule's input description. In counterbleeding, a later rule removes part of the input description of an earlier rule, such that the earlier rule seems, on the surface, to have applied without satisfying its input description.

For example, if rule $\mathcal{F}$ from above is ordered after $\mathcal{R}$, giving rise to a counterbleeding on environment rule interaction, then the input / rartər/ is mapped by $\mathcal{R}$ to rsitər, which $\mathcal{F}$ maps to [rıIrər]. $\mathcal{R}$ is then opaque in the sense of (ii): $[\mathrm{AI}]$ occurs in an environment other than _ $\mathrm{t}$. In such a case, I will often abstract away from the rules and say that the input-output pattern /rartər/ $\rightarrow$ [rıIrər] is opaque.

Although opacity is defined here in terms of rules, a natural question is whether opaque input-output patterns like /rartər/ $\rightarrow$ [rıIrər] are expressible by other formalisms, like OT grammars. In the next section, I describe the architecture of OT grammars, for which such patterns have been problematic. 


\begin{tabular}{|c|c|c|c|c|}
\hline /raitər / & *aIt & *VtV & ID (low) & ID (son) \\
\hline a. $\quad$ raitər & 1 & 1 & 0 & 0 \\
\hline b. rsitər & 0 & 1 & 1 & 0 \\
\hline c. rairər & 0 & 0 & 0 & 1 \\
\hline d. rıIrər & 0 & 0 & 1 & 1 \\
\hline
\end{tabular}

Fig. 1. An example OT tableau.

\subsection{Optimality Theoretic Phonology}

In an OT grammar, an input like / raitər/ is first fed into a function GEN that generates an infinite set of output candidates. In practice, every possible output is a candidate. This set is then filtered down via a potentially infinite set of strictly, totally ordered constraints until a unique output candidate remains. The entire process can be visualized using an OT tableau.

For example, Fig. 1 presents a tableau with four output candidates for the input / raitər/ and four constraints, ranked left to right from highest to lowest. The process begins with the leftmost constraint, *art, which is an output-markedness constraint that assigns a violation for each occurrence of the sequence [art] in an output candidate. Candidate (a) thus incurs a violation of 1 , while the other candidates incur 0 violations; hence, candidate (a) is eliminated. The next constraint, ${ }^{*} \mathrm{VtV}$, which assigns a violation mark for each intervocalic [t] in an output candidate, eliminates candidate (b). Next, IDENT-IO(low), an input-output faithfulness constraint which penalizes each occurrence of a low vowel like /aI/ mapping to a non-low vowel like $[\Lambda \mathrm{I}]$, eliminates candidate $(\mathrm{d})$. At this point, only candidate (c) remains and is therefore the optimal output. The last constraint, IDENT-IO(sonorant), which penalizes mapping a non-sonorant segment like /t/ to a sonorant one like [r], effectively does no work here.

Note that in this tableau the opaque input-output pattern / raitər $/ \rightarrow[\mathrm{r} \Lambda$ Irər $]$ is not optimal. Moreover, the violations assigned to [r $\mathrm{r}$ Irər] are a proper superset of those assigned to [rairər]. Consequently, no reranking of these particular constraints can possibly make [rıIrər] more optimal than [raIrər]. This is the sort of reasoning that underlies the intuition that opacity is problematic for OT.

I now give a formal characterization of OT grammars, following [8,17]. If inputs and output candidates are taken to be strings of symbols over $\Sigma$, then GEN is a relation over $\Sigma^{*} \times \Sigma^{*}$ : it pairs input strings with (sets of) output candidate strings. ${ }^{8}$ An OT grammar can then be defined formally as follows.

Definition 7. An OT grammar is a tuple $\left\langle\Sigma\right.$, GEN, $\left.C,>_{C}\right\rangle$, where GEN $\subseteq \Sigma^{*} \times$ $\Sigma^{*} ; C$ is a set of functions from $\mathrm{GEN}$ to $\mathbb{N}$; and $>_{C}$ is a strict, total order on C.

A relation $>_{\mathcal{G}}$ over GEN defines optimality relative to two input-output pairs: $\langle i, o\rangle>_{\mathcal{G}}\left\langle i, o^{\prime}\right\rangle$ means that $\langle i, o\rangle$ is more optimal than $\left\langle i, o^{\prime}\right\rangle$ in the OT grammar

\footnotetext{
${ }^{8}$ Recall that $\Sigma^{*}$ denotes the set of all strings over the symbols in $\Sigma$, including the empty string.
} 
$\mathcal{G}$. In such a case, I will often write that $o$ is more optimal than $o^{\prime}$ with respect to $i$ (in $\mathcal{G}$ ).

Definition 8. Given an $O T$ grammar $\mathcal{G}=\left\langle\Sigma\right.$, GEN $\left., C,>_{C}\right\rangle$, with $\langle i, o\rangle,\left\langle i, o^{\prime}\right\rangle$ $\in$ GEN, $\langle i, o\rangle>_{\mathcal{G}}\left\langle i, o^{\prime}\right\rangle$ iff there is some $c_{j} \in C$ such that $c_{j}(\langle i, o\rangle)\left\langle c_{j}\left(\left\langle i, o^{\prime}\right\rangle\right)\right.$, and for each $c_{k}$ such that $c_{k}>_{C} c_{j}, c_{k}(\langle i, o\rangle)=c_{k}\left(\left\langle i, o^{\prime}\right\rangle\right)$.

A single output candidate for some input is then optimal just in case it is more optimal than every other candidate with respect to that input.

Definition 9. Given an $O T$ grammar $\left.\mathcal{G}=\langle\Sigma \text {, GEN, } C,\rangle_{C}\right\rangle,\langle i, o\rangle \in$ GEN is optimal in $\mathcal{G}$ iff for each $\left\langle i, o^{\prime}\right\rangle \in$ GEN (o different from $o^{\prime}$ ), $\left.\langle i, o\rangle\right\rangle_{\mathcal{G}}\left\langle i, o^{\prime}\right\rangle$.

Turning now to the constraint set, an OT constraint can be represented by a weighted finite state transducer (FST) $[8,9,7]$, called a finite state OT constraint. This FST essentially "reads" or "processes" pairs of input-output strings, one input-output symbol pair at a time, transitioning from state to state and assigning 0 or 1 violations to each pair of corresponding input-output symbols. The symbol $\epsilon$ here denotes the empty string. (It should not be confused with the IPA symbol $\varepsilon$, which denotes an open-mid front vowel.)

Definition 10. A finite state OT constraint is a tuple $\left\langle Q, \Sigma, \delta, q_{0}, F\right\rangle$, where:

1. $Q$ is a non-empty set of states;

2. $\Sigma$ is a set of symbols;

3. $\delta$ is a transition function from $Q \times \Sigma^{\epsilon} \times \Sigma^{\epsilon}$ to $\{0,1\} \times Q$, where $\Sigma^{\epsilon}=\Sigma \cup\{\epsilon\}$;

4. $q_{0} \in Q$ is the unique start state;

5. $F \subseteq Q$ is the non-empty set of final states.

The transition function $\delta$ takes in a triple $\left\langle q_{j}, i, o\right\rangle$ consisting of some initial state $q_{j} \in Q$, an input symbol $i \in \Sigma^{\epsilon}$, and an output symbol $o \in \Sigma^{\epsilon}$ and returns a pair $\left\langle n, q_{k}\right\rangle$ consisting of some number of violations $n \in\{0,1\}$ and some terminal state $q_{k} \in Q$. For ease of exposition, I will write members of $\delta$ as $\left\langle q_{j}, i, o, n, q_{k}\right\rangle$ instead of $\left\langle\left\langle q_{j}, i, o\right\rangle,\left\langle n, q_{k}\right\rangle\right\rangle$ and say that $\delta$ assigns $n$ violations to the pair $i \rightarrow o$ (on the transition from $q_{j}$ to $q_{k}$ ).

Consider, for example, the faithfulness constraint IDENT-IO(low), which, to repeat, penalizes each mapping of a low vowel like /aI/ to a non-low vowel like $[\Lambda \mathrm{I}]$. I assume that diphthongs like $[\mathrm{aI}]$ and $[\Lambda \mathrm{I}]$ are single units rather than segments composed of two vowels. Formally, this means ar and $\Lambda \mathrm{I}$ are each single symbols in $\Sigma$. IDENT-IO(low) can then be represented by the single-state FST

$$
\operatorname{id}(\text { low })=\left\langle\left\{\operatorname{id}(\text { low })_{0}\right\},\{\text { ar }, \Lambda \mathrm{I}, \ldots\}, \delta, \operatorname{id}(\text { low })_{0},\left\{\operatorname{id}(\text { low })_{0}\right\}\right\rangle
$$

where $\delta$ assigns 1 to the pair aI $\rightarrow \Lambda \mathrm{I}$ and 0 to every other pair. This FST is conventionally visualized as in Fig. 2. Thus, an input-output pair like $\langle$ rartər, rartər $\rangle$ incurs 0 total violations, whereas $\langle$ rartər, rsitər $\rangle$ incurs 1 total violation.

A markedness constraint like *art, which penalizes each occurrence of the sequence [art] in an output candidate, can be represented by the two-state FST

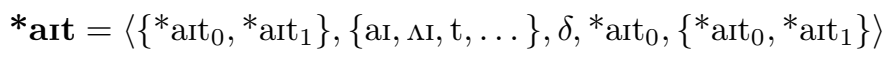




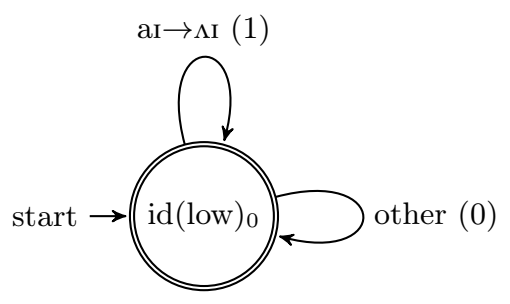

Fig. 2. A visualization of id(low).

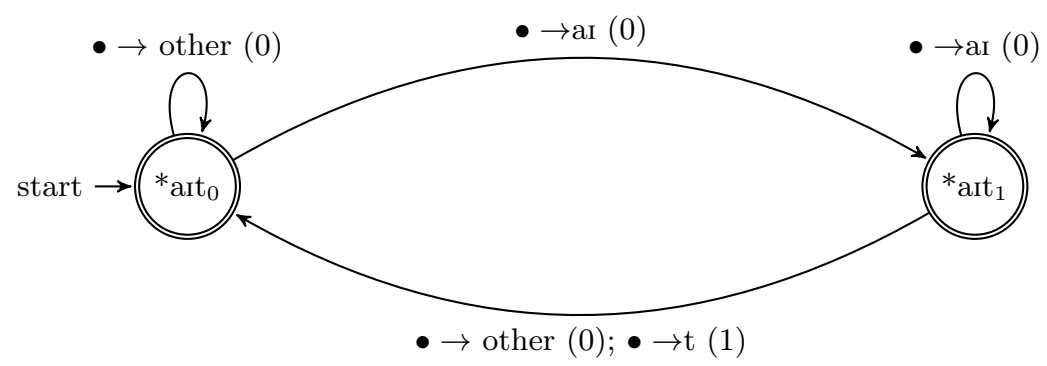

Fig. 3. A visualization of *art.

where $\delta$ assigns 1 whenever the sequence [art] occurs in an output, regardless of the input, and 0 otherwise. This FST is visualized as in Fig. 3, where $\bullet$ stands for any symbol from the symbol set (or the empty string).

Of note is that the violations assigned by a markedness FST are independent of the input (hence the $\bullet$ on every transition). By contrast, the violations assigned by a faithfulness FST depend on the input. This can be formalized as follows. ${ }^{9}$

Definition 11. Given an OT grammar $\mathcal{G}=\left\langle\Sigma\right.$, GEN $\left., C,>_{C}\right\rangle$, a constraint $c \in$ $C$ is a markedness constraint iff it can be represented by a finite state constraint $\left\langle Q, \Sigma, \delta, q_{0}, F\right\rangle$ such that, if $\left\langle q_{j}, \alpha, \beta, 1, q_{k}\right\rangle \in \delta$, then for any $\gamma \in \Sigma^{\epsilon}$ such that $\left\langle q_{j}, \gamma, \beta, n, q_{k}\right\rangle \in \delta$, it follows that $n=1$. An FST that represents a markedness constraint is a finite state markedness constraint.

Definition 12. Given an $O T$ grammar $\mathcal{G}=\left\langle\Sigma\right.$, GEN $\left., C,>_{C}\right\rangle$, a constraint $c \in$ $C$ is a faithfulness constraint iff it can be represented by a finite state constraint $\left\langle Q, \Sigma, \delta, q_{0}, F\right\rangle$ such that $\left\langle q_{j}, \alpha, \beta, 1, q_{k}\right\rangle \in \delta$ and $\left\langle q_{j}, \gamma, \beta, 0, q_{k}\right\rangle \in \delta$, for some $\alpha, \beta, \gamma \in \Sigma^{\epsilon}$ and for some $q_{j}, q_{k} \in Q$. An FST that represents a faithfulness constraint is a finite state faithfulness constraint.

Moreover, whereas (finite state) markedness constraints may have one or more states, standard faithfulness constraints like IDENT, MAx, and DeP have

\footnotetext{
${ }^{9}$ When there is no confusion, I will write "markedness (faithfulness) constraint" rather than "finite state markedness (faithfulness) constraint" to refer to the FST representing some OT constraint.
} 
just one state: when assigning a violation mark to an input-output symbol pair $a \rightarrow b$, these constraints do not "look ahead" or "look behind" at other symbols in the input or output. I call this sort of constraint a single-state faithfulness constraint.

Definition 13. Given an $O T$ grammar $\mathcal{G}=\left\langle\Sigma\right.$, GEN $\left., C,>_{C}\right\rangle$, a constraint $c \in$ $C$ is a single-state faithfulness constraint iff it can be represented by a finite state faithfulness constraint $\left\langle Q, \Sigma, \delta, q_{0}, F\right\rangle$ such that $|Q|=1$.

The definition of a classic OT grammar can now be stated as follows. ${ }^{10,11}$

Definition 14. A classic OT grammar is an OT grammar $\mathcal{G}=\left\langle\Sigma\right.$, Gen, $\left.C,>_{C}\right\rangle$ such that (i) if $\langle i, o\rangle \in \mathrm{GEN}$, then $\left\langle i, o^{\prime}\right\rangle \in \mathrm{GEN}$, for each $o^{\prime} \in \Sigma^{*}$; and (ii) each $c \in C$ is either is a markedness constraint or a single-state faithfulness constraint.

\section{The Proof}

In this section I prove the following claim.

Claim. There is a relation $R$ such that (i) $R$ is regular, and (ii) there is no classic OT grammar $\mathcal{G}$ such that each $\langle i, o\rangle \in R$ is optimal in $\mathcal{G}$.

The patterns I will use are the Canadian raising patterns already discussed, presented in Fig. 4. I first show that these patterns are expressible by ordered rewrite rules; hence, they are regular, proving part (i) of the claim. I then show, less trivially, that the patterns cannot be expressed by any classic OT grammar, proving part (ii) of the claim. The main result is proved as Theorem 2.

All four patterns in Fig. 4 can be captured by a rule $\mathcal{R}$ of raising /aI/ to [ $\Lambda \mathrm{I}]$ before underlying /t/

$$
\mathcal{R}: \text { aI } \rightarrow \Lambda \mathrm{I} / \text { t }
$$

ordered before a rule $\mathcal{F}$ of flapping (changing intervocalic /t/ or /d/ to [r])

$$
\mathcal{F}: \mathrm{t}, \mathrm{d} \rightarrow \mathrm{r} / \mathrm{V}-\mathrm{V}
$$

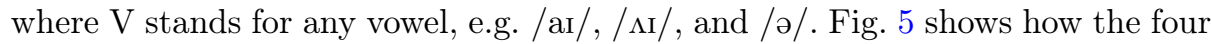
patterns are expressed by the interaction of these two rules.

${ }^{10}$ The requirement on GEN in (i) is intended to capture the assumption, stated above, that every possible output of a given input is considered to be a candidate. See the discussion of freedom of analysis in [16, p. 20]. This requirement also ensures that the relevant candidates are in competition in order for the proof in section 3 to proceed, though any weaker requirement that ensures this would suffice, too.

${ }^{11}$ Some phonologists might object that the single-state restriction on faithfulness constraints in (ii) is not enough, since, for example, faithfulness constraints penalizing arbitrary input-output symbols are allowed. However, if, as I prove in section 3, even this relatively powerful version of classic OT is incapable of expressing certain regular relations, then so is any more restrictive version of it. 


\begin{tabular}{|c|c|c|c|c|}
\hline & \multicolumn{2}{|l|}{ Input } & \multicolumn{2}{|l|}{ Output } \\
\hline a. & /rait/ & $\rightarrow$ & {$[\mathrm{r} \Lambda \mathrm{It}]$} & "write" \\
\hline b. & /raid/ & $\rightarrow$ & [raId] & "ride" \\
\hline c. & /raitər / & $\rightarrow$ & {$[\mathrm{r} \Lambda \mathrm{Ir} \partial \mathrm{r}]$} & "writer" \\
\hline d. & /raidər/ & $\rightarrow$ & [rairər] & "rider" \\
\hline
\end{tabular}

Fig. 4. Canadian raising data.

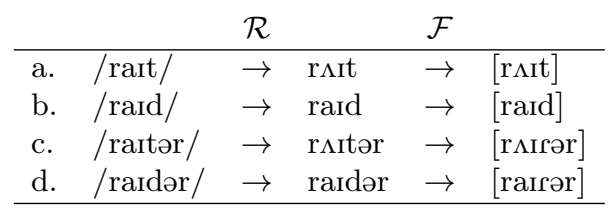

Fig. 5. Rule-based derivations of Canadian raising.

Recall that the pattern /rartər/ $\rightarrow$ [rıIrər] is the crucial opaque pattern: /ar/ raises to $[\Lambda \mathrm{I}]$ because of the underlying / $\mathrm{t} /(\mathcal{R}$ 's environment), but $/ \mathrm{t} /$ is changed to $[r]$ by $\mathcal{F}$. Thus, $\mathcal{F}$ removes the environment that motivates the application of $\mathcal{R}$, obscuring, on the surface, the reason for raising.

These patterns are intuitively problematic for classic OT for the following reason: given the evidence from /raidər/ that [rairər] is an unmarked output, it is always more optimal to map /rartər/ to [rairər], incurring just one faithfulness violation $(\mathrm{t} \rightarrow \mathrm{r})$, than to map / raitər/ to [rıIrər], incurring two faithfulness violations $(t \rightarrow r$, aI $\rightarrow \Lambda \mathrm{I})$. (This explains why in Fig. 1 of the previous section,

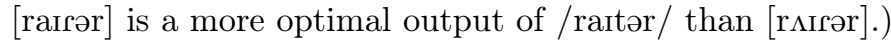

Informally, the patterns /raitər/ $\rightarrow$ [rıIrər] and /raIdər $/ \rightarrow$ [raIrər] are conflicting. More formally, we have the following theorem.

Theorem 1. Let $\left.\mathcal{G}=\langle\Sigma, \mathrm{GEN}, C,\rangle_{C}\right\rangle$ be a classic OT grammar such that

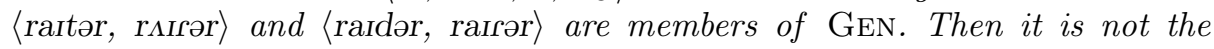

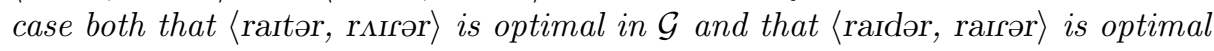
in $\mathcal{G}$.

Proof. The proof is by contradiction. Assume both that $\langle$ rartər, rsirər $\rangle$ is optimal in $\mathcal{G}$ and that $\langle$ raIdər, raIrər $\rangle$ is optimal in $\mathcal{G}$. Then for each $\langle$ rartər, o $\in \in$ GEN (with $o$ different from [rıIrər]), $\langle$ raitər, r $\Lambda I r ə r\rangle>_{\mathcal{G}}\langle$ raitər, $o\rangle$; and for each $\langle$ raIdər, $o\rangle \in$ GEN (with $o$ different from [raIrər]), $\langle$ raIdər, raIґər $\rangle>_{\mathcal{G}}\langle$ raIdər, $o\rangle$.

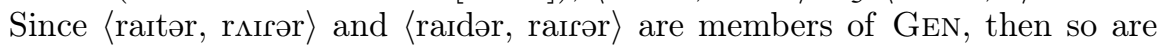
$\langle$ raitər, raIrər $\rangle$ and $\langle$ raIdər, rsIrər $\rangle$. It follows both that $\langle$ raitər, rsIrər $\rangle>_{\mathcal{G}}\langle$ raitər, raIrər $\rangle$ and that $\langle$ raIdər, raIґər $\rangle>_{\mathcal{G}}\langle$ raIdər, r $\Lambda$ Irər $\rangle$. By the definition of optimality, the following statements are derived.

There is a $c_{j} \in C$ such that $c_{j}(\langle$ raitər, rıIгər $\rangle)<c_{j}(\langle$ raitər, raIrər $\rangle)$.

For all $c^{\prime} \in C$ such that $\left.c^{\prime}\right\rangle_{C} c_{j}, c^{\prime}(\langle$ rartər, ruIrər $\rangle)=c^{\prime}(\langle$ rartər, raIґər $\rangle)$.

There is a $c_{k} \in C$ such that $c_{k}(\langle$ raidər, raIrər $\rangle)<c_{k}(\langle$ raIdər, rsIгər $\rangle)$.

For all $c^{\prime} \in C$ such that $\left.c^{\prime}\right\rangle_{C} c_{k}, c^{\prime}(\langle$ raIdər, raI əər $\rangle)=c^{\prime}(\langle$ raIdər, rıIґər $\rangle)$. 
Recall that, as defined here, classic OT grammars contain only markedness and single-state faithfulness constraints. Thus, to prove the theorem, it must be shown that at least one of $c_{j}, c_{k}$ is neither of these types. There are four cases to consider.

CASE 1. Suppose that both $c_{j}$ and $c_{k}$ are markedness constraints. Then $c_{j}$ assigns $m$ violation marks to [rیIrər] and $n$ violations to [raIrər], regardless of input; hence, from (1) it follows that $m<n$. Similarly, $c_{k}$ assigns $p$ violation marks to [ruIrər] and $q$ violations to [raIrər], regardless of input; hence, from (3) it follows that $q<p$.

It cannot be the case that $c_{j}$ and $c_{k}$ are the same constraint, for then $m=p$ and $n=q$, deriving a contradiction. Thus, $c_{j}$ and $c_{k}$ are different constraints, with one ranked above the other. Suppose that $c_{j}>_{C} c_{k}$. Then it follows from (4) that

$$
c_{j}(\langle\text { raidər, rairər }\rangle)=c_{j}(\langle\text { raidər, } \mathrm{r \Lambda Irər}\rangle)
$$

and hence $n=m$. But it was already established that $m<n$, so it cannot be that $c_{j}>_{C} c_{k}$.

Now suppose that $c_{k}>_{C} c_{j}$. Then it follows from (2) that

$$
c_{k}(\langle\text { rartər, } \mathrm{r \Lambda I \digamma ər}\rangle)=c_{k}(\langle\text { raitər, raiґər }\rangle)
$$

and hence $p=q$. But it was already established that $q<p$, so it cannot be that $c_{k}>_{C} c_{j}$. We have reached a contradiction, so $c_{j}$ and $c_{k}$ cannot both be markedness constraints. At least one of them is a single-state faithfulness constraint.

CASE 2. Suppose that $c_{j}$ is a single-state faithfulness constraint and that $c_{k}$ is a markedness constraint. From (1) it follows that the FST representing $c_{j}$ assigns 1 violation to the pair aI $\rightarrow$ ar and 0 violations to the pair aI $\rightarrow \Lambda \mathrm{I}$, because the two inputs are the same and the two output candidates differ only in having [aI] or $[\Lambda \mathrm{I}]$ as the second segment. Thus, it follows that

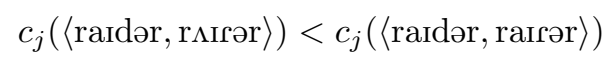

since, again, the two inputs are the same and the two output candidates differ only in the second segment.

Since $c_{j}$ and $c_{k}$ are different constraints, one must outrank the other. It cannot be that $c_{j}>_{C} c_{k}$, for it was just established that

$$
c_{j}(\langle\text { raidər, rıIґər }\rangle) \neq c_{j}(\langle\text { raidər, raIґər }\rangle)
$$

which contradicts (4). Suppose, then, that $c_{k}>_{C} c_{j}$. Since by hypothesis $c_{k}$ is a markedness constraint, then from (3) it follows that $c_{k}$ assigns fewer violations to [raIrər] than to [ruIrər], regardless of input, so that

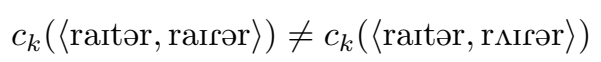

But this contradicts (2). We have reached a contradiction: if $c_{j}$ is a single-state faithfulness constraint, then $c_{k}$ cannot be a markedness constraint. 
CASE 3. Suppose that $c_{j}$ and $c_{k}$ are both single-state faithfulness constraints. From (3) it follows that the FST representing $c_{k}$ assigns 1 violation to the pair aI $\rightarrow \Lambda$ I and 0 violations to the pair aI $\rightarrow$ aI. Thus, it follows that

$$
c_{k}(\langle\text { raitər, rairər }\rangle)<c_{k}(\langle\text { raitər, r } \Lambda \text { Irər }\rangle)
$$

Since this statement is different from (1), $c_{j}$ and $c_{k}$ must be different constraints and hence ranked one over the other. It cannot be that $c_{k}>_{C} c_{j}$, for the above statement contradicts (2). However, as was established in case 2 , if $c_{j}$ is a singlestate faithfulness constraint, then it cannot be that $c_{j}>_{C} c_{k}$. We have reached a contradiction: if $c_{k}$ is a single-state faithfulness constraint, then $c_{j}$ must be a markedness constraint.

CASE 4. Suppose that $c_{j}$ is a markedness constraint and that $c_{k}$ is a singlestate faithfulness constraint. From (1) it follows that $c_{j}$ assigns fewer violations to [rıIгər] than to [raIґər], regardless of input; hence, from the statement derived in case 3 concerning $c_{k}$ as a single-state faithfulness constraint, it follows that $c_{j}$ and $c_{k}$ are different constraints, with one ranked above the other.

It cannot be that $c_{j}>_{C} c_{k}$, for otherwise

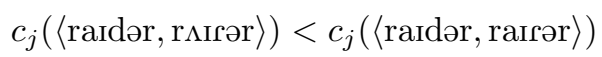

which contradicts (4). Thus, $c_{k}>_{C} c_{j}$. However, as already established in case 3 , if $c_{k}$ is a single-state faithfulness constraint, then it cannot be that $c_{k}>_{C} c_{j}$.

We have reached a contradiction and have exhausted all cases: there are no markedness or single-state faithfulness constraints that satisfy (1-4). Hence, it

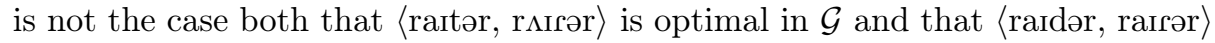
is optimal in $\mathcal{G}$.

From Theorem 1, together with the rule-based analysis above, the claim at the start of this section follows easily.

Theorem 2. There is a relation $R$ such that (i) $R$ is regular, and (ii) there is no classic OT grammar $\mathcal{G}$ such that each $\langle i, o\rangle \in R$ is optimal in $\mathcal{G}$.

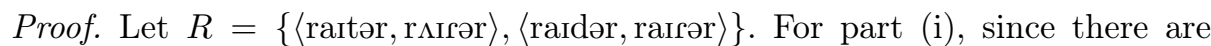

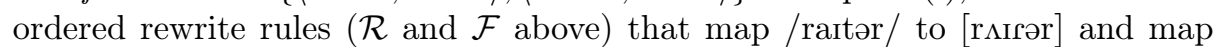
/raIdər/ to [raIrər], it follows that $R$ is regular. Part (ii) follows from Theorem 1.

\section{Discussion}

In the previous section, I proved that Canadian raising, which is an example of counterbleeding on environment opacity, is expressible by ordered rewrite rules but not by classic OT grammars. I now show that there are other examples of counterbleeding on environment opacity, as well as examples of counterfeeding 


\begin{tabular}{|c|c|c|c|c|}
\hline & Input & & Output & \\
\hline a. & $/ \mathrm{sol} /$ & $\rightarrow$ & {$[\mathrm{sul}]$} & "rubble" \\
\hline b. & /gruz/ & $\rightarrow$ & [grus] & "salt" \\
\hline c. & /3wob/ & $\rightarrow$ & [3wup] & "crib" \\
\hline d. & /3wop/ & $\rightarrow$ & [3wop] & hypothetical \\
\hline
\end{tabular}

Fig. 6. Polish data.

\begin{tabular}{|c|c|c|c|}
\hline Input & & Output & \\
\hline /ha:kimi:n/ & $\rightarrow$ & [ha:k $\left.\mathrm{k}^{\mathrm{j}} \min \right]$ & "ruling (masc. pl.) \\
\hline /ha:kminn/ & $\rightarrow$ & [ha:kmi:n] & hypothetical \\
\hline
\end{tabular}

Fig. 7. Bedouin Arabic data 1.

on environment opacity, that are likewise inexpressible by classic OT grammars. Though the patterns are all different, I discuss several formal properties that they share. ${ }^{12}$

\subsection{Other Examples of Counterbleeding on Environment Opacity}

Polish. Consider the data from Polish in Fig. 6 [18,19,12]. All four patterns can be captured by a rule $\mathcal{R}$ of raising /o/ to [u] before voiced segments $(/ \mathrm{l}, \mathrm{b} /)$, ordered before a rule $\mathcal{D}$ of devoicing word-final obstruents, i.e. mapping wordfinal /z, b/ to $[\mathrm{s}, \mathrm{p}]$. $\mathcal{D}$ counterbleeds on $\mathcal{R}$ 's environment because $\mathcal{D}$ removes part of $\mathcal{R}$ 's environment $\left(\_\mathrm{l}, \mathrm{b}\right)$ by mapping $/ \mathrm{b} /$ to $[\mathrm{p}]$.

The patterns /3wob/ $\rightarrow$ [3wup] and /3wop/ $\rightarrow$ [3wop] cannot be expressed by any classic OT grammar. The proof is essentially the same as that of Theorem 1 , and the reason is that the Canadian raising patterns and the Polish patterns are formally almost identical. In Canadian raising, /aI/ surfaces as $[\Lambda \mathrm{I}]$ before / $\mathrm{t} /$, which (along with /d/) surfaces as [r]. In Polish, /o/ surfaces as [u] before /b/, which (along with $/ \mathrm{p} /$ ) surfaces as $[\mathrm{p}]$. The difference is that in Canadian raising, /t, d/ surface as a third segment, [r], whereas in Polish, /b, p/ surface as [p].

Bedouin Arabic 1. Consider the data from Bedouin Arabic in Fig. 7 [11]. Both patterns are expressible by a rule $\mathcal{P}$ of palatalizing $/ \mathrm{k} /$ to $\left[\mathrm{k}^{\mathrm{j}}\right]$ before $/ \mathrm{i} /$, ordered before a rule $\mathcal{D}$ of deleting /i/ (mapping /i/ to $\epsilon$ ). $\mathcal{D}$ counterbleeds on $\mathcal{P}$ 's environment because $\mathcal{D}$ removes part of $\mathcal{P}$ 's environment ( $\_$i) by deleting /i/. These two patterns cannot be expressed by any classic OT grammar, and the reason, again, is that these patterns are formally similar to those in Canadian raising: / $\mathrm{k} /$ surfaces as $\left[\mathrm{k}^{\mathrm{j}}\right]$ before $/ \mathrm{i} /$, which (along with $\epsilon$ ) surfaces as $\epsilon$.

${ }^{12}$ In each of the following examples, I employ a hypothetical input that is not actually part of that language's lexicon, as far as I know. Nonetheless, I take such absences to simply be accidental lexical gaps. Moreover, the formal result that these sets of input-outputs patterns are expressible by ordered rules but not by classic OT still holds; the empirical question of whether the patterns are attested is a separate issue. 


\begin{tabular}{|c|c|c|c|c|}
\hline & Input & & Output & \\
\hline a. & /támi/ & $\rightarrow$ & [tám] & "it ends" \\
\hline b. & /tájo:l/ & $\rightarrow$ & [tájo:l] & "shelled corn" \\
\hline c. & / Sikakíli/ & $\rightarrow$ & [ jikakíl] & "put it in it" \\
\hline d. & / Sikakíl/ & $\rightarrow$ & 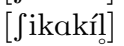 & hypothetical \\
\hline
\end{tabular}

Fig. 8. Isthmus Nahuat data.

\begin{tabular}{lllll}
\multicolumn{2}{c}{ Input } & \multicolumn{2}{c}{ Output } \\
\hline a. $/$ gabr $/ \rightarrow$ & [gabur] & "grave" \\
b. & gabur $/ \rightarrow$ & ggibur $]$ & hypothetical \\
\hline
\end{tabular}

Fig. 9. Bedouin Arabic data 2.

\subsection{Examples of Counterfeeding on Environment Opacity}

Isthmus Nahuat. Consider the data from Isthmus Nahuat in Fig. 8 [20,19,16]. All four patterns are expressible by a rule $\mathcal{D}$ of devoicing /l/ to [1] word-finally, ordered before a rule $\mathcal{A}$ of apocope, i.e. deleting /i/ word-finally. $\mathcal{A}$ counterfeeds on $\mathcal{D}$ 's environment because $\mathcal{A}$ creates part of $\mathcal{D}$ 's environment (_ \#, where \# denotes a word boundary) by deleting a word-final /i/ (mapping i\# to $\epsilon \#$ ).

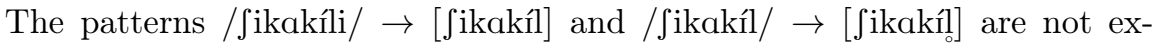
pressible by any classic OT grammar. The proof, omitted here, is essentially the same as that of Theorem 1 for counterbleeding on environment opacity, using

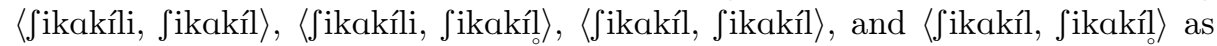
the relevant members of GEN. Informally, the reason that these patterns are

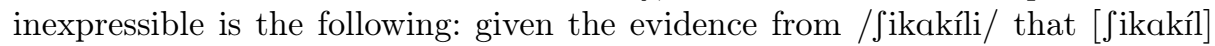

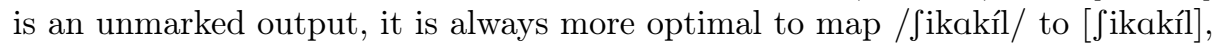

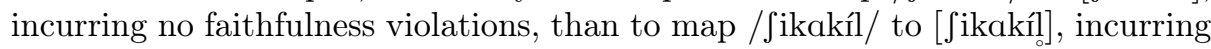
one faithfulness violation $(1 \rightarrow 1)$. Or, in terms of the formalism presented here, /i/ surfaces as $\epsilon$ word-finally, and /l/ surfaces as [1], but only if /l/ does not precede a word-final /i/; and this latter condition on the identity of $/ 1 /$ can be expressed only by a multistate faithfulness constraint.

Bedouin Arabic 2. Fig. 9 presents more data from Bedouin Arabic [11]. Both patterns can be captured by a rule $\mathcal{R}$ of raising /a/ to [i] in an open syllable, ordered before a rule $\mathcal{E}$ of epenthesis, i.e. inserting a $[\mathrm{u}]$ (mapping $\epsilon$ to $[\mathrm{u}]$ ) between two consonants like $/ \mathrm{br} / . \mathcal{E}$ counterfeeds on $\mathcal{R}$ 's environment because $\mathcal{E}$ creates part of $\mathcal{R}$ 's environment $(\ldots \mathrm{bV})$ by inserting a vowel, [i], after [b]. These two patterns cannot be expressed by any classic OT grammar, and the reason is that these patterns are formally similar to those in Isthmus Nahuat. In Bedouin Arabic, $\epsilon$ surfaces as [u] between /br/, and /a/ surfaces as [i], but only when /a/ does not precede a consonant cluster.

Lomongo. Consider, finally, the data from Lomongo in Fig. 10 [21,19,12]. Both patterns can be captured by a rule $\mathcal{G}$ of prevocalic gliding, i.e. mapping /o/ to 


\begin{tabular}{lllll}
\multicolumn{2}{c}{ Input } & \multicolumn{2}{c}{ Output } & \\
\hline a. & /obina/ & $\rightarrow$ & oina $]$ & "you (sg.) dance" \\
b. & /oina/ & $\rightarrow$ & [wina $]$ & hypothetical \\
\hline
\end{tabular}

Fig. 10. Lomongo data.

\begin{tabular}{|c|c|c|c|c|}
\hline Language & \multicolumn{2}{|c|}{ Inputs } & \multicolumn{2}{|c|}{ Outputs } \\
\hline Canadian English & raitər & raIdər & rairər & 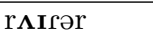 \\
\hline Polish & 3wob & 3wop & 3wop & 3wup \\
\hline Bedouin Arabic 1 & ha:kimi:n & ha:kemi:n & ha:k $\epsilon$ mi:n & $\hbar a: \mathbf{k}^{\mathbf{j}} \in \operatorname{mi}: n$ \\
\hline Isthmus Nahuat & fikakíli & 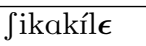 & \ikakíl $\epsilon$ & \ikakíl $\epsilon$ \\
\hline Bedouin Arabic 2 & gaber & gabur & gabur & gibur \\
\hline Lomongo & obina & oєina & $\mathbf{o} \epsilon$ ina & $\mathbf{w} \epsilon$ ina \\
\hline
\end{tabular}

Fig. 11. A summary of all the input-output candidate pairs in this paper.

[w] before a vowel, ordered before a rule $\mathcal{D}$ of intervocalic obstruent deletion, i.e. deleting /b/ between two vowels. $\mathcal{D}$ counterfeeds on $\mathcal{G}$ 's environment because $\mathcal{D}$ creates part of $\mathcal{G}$ 's environment $(-\mathrm{V})$ by deleting a prevocalic /b/, i.e. by mapping /bV/ to just [V]. These patterns are not expressible by any classic OT grammar, and the reason, once more, is that these patterns are similar to those in Isthmus Nahuat. In this case, /b/ surfaces as $\epsilon$ between two vowels, and /o/ surfaces as [w], but only when /o/ does not precede a prevocalic /b/.

Summary. Fig. 11 summarizes all of the sets of input-output candidate pairs that have been discussed in this paper, with the empty string symbol $(\epsilon)$ included where appropriate. Each of the six sets exhibits the following three properties: (i) the two inputs differ by exactly one segment $x$; (ii) the two output candidates differ by exactly one segment $y$; and (iii) $x$ is different from $y$, i.e. they occupy different string positions. (In Fig. 11, the differing segments are in bold.) Formally, then, these sets are essentially the same; hence, it is not surprising that in all six examples the relevant input-output patterns cannot be made optimal by classic OT grammars, as defined here. Moreover, from this perspective it emerges that the difference between the counterbleeding on environment examples and the counterfeeding on environment examples boils down to which two (of the four possible) input-output candidate pairs are supposed to be optimal.

Notably, the proof technique used in this paper (finding two conflicting inputoutput patterns) does not seem to work for cases of focus opacity. To give just one example of counterfeeding on focus opacity, in Western Basque [22], /a/ raises to $[\mathrm{e}]$, and /e/ raises to [i], but /a/ does not raise to [i]. To express the latter generalization in OT, it suffices to posit an undominated, single-state faithfulness constraint that assigns 1 to the pair a $\rightarrow$ i and 0 to every other pair. Such a constraint may seem intuitively ad hoc, yet it is formally sound.

More generally, the reason that focus opacity seems unproblematic for OT from this paper's perspective is twofold: (i) classic faithfulness constraints, as defined here, are single-state faithfulness constraints that can penalize arbitrary, 
single input-output segment pairs; and (ii) in focus opacity, all change occurs at a single focus. Thus, it suffices to posit a single-state faithfulness constraint that assigns 1 violation to those focus changes that are undesired, like $a \rightarrow i$, and 0 to those that are desired. Moreover, taking into account Fig. 11 and the three properties of environment opacity mentioned above, it comes as no surprise that the focus opacity of Western Basque works differently: it necessarily lacks at least one of the three properties. Specifically, if the first two properties hold, i.e. the relevant inputs differ by exactly one segment $x$ and the relevant output candidates differ by exactly one segment $y$, then the third property, according to which $x$ and $y$ occupy different string positions, must not hold, because in focus opacity the segments that change (a, e, i) all occupy the same string position.

\section{Conclusion}

In this paper I defined a classic OT grammar as any OT grammar that contains only markedness constraints, i.e. constraints representable by an FST that is input-independent, and single-state faithfulness constraints, i.e. constraints representable by an FST that is both input-dependent and single-state. I then proved, using data from Canadian English, that there are input-output patterns which can be expressed by SPE-style, ordered, context-sensitive rewrite rules, but which cannot be expressed by any classic OT grammar (Theorem 1). Hence, there are regular relations that classic OT grammars, as defined here, cannot express (Theorem 2). I also demonstrated that several other cases of counterbleeding on environment opacity, as well as several cases of counterfeeding on environment opacity, are likewise expressible by ordered rewrite rules but not by classic OT. Lastly, I argued that focus opacity, unlike environment opacity, seems unproblematic for classic OT grammars that allow arbitrary single-state faithfulness constraints.

Regarding empirical coverage, assuming that the Canadian raising data are attested, then classic OT, as defined here, undergenerates. Assuming also that natural language phonology is strictly subregular (see [5]), then, since I did not place any upper bound on the number of violations that any constraint may assign, classic OT also overgenerates: it can express unattested, non-regular patterns (see footnote 3). Ordered rewrite rules, under the same assumptions, only overgenerate. ${ }^{13}$ However, whether or not this serves as a basis to favor rules over classic OT remains to be seen: it could be, for example, that the classes of patterns by which classic OT grammars over- and undergenerate are in some formal sense smaller (or more manageable) than the class of patterns by which rules overgenerate.

\section{Acknowledgments}

I thank Morgan Sonderegger for his supervision at every stage of this research; Brendan Gillon, Heather Goad, and Michael Wagner for helpful discussions; and

\footnotetext{
${ }^{13}$ I thank an anonymous referee for raising these points.
} 
three anonymous referees for Formal Grammar and two anonymous referees for Mathematics of Language for their comments, which included pointing out an incorrect claim in an earlier version of this paper.

\section{References}

1. Chomsky, N., Halle, M.: The Sound Pattern of English. The MIT Press (1968)

2. Prince, A., Smolensky, P.: Optimality Theory: Constraint Interaction in Generative Grammar. Blackwell Publishers (2004)

3. Johnson, C.D.: Formal Aspects of Phonological Description. Mouton (1972)

4. Kaplan, R.M., Kay, M.: Regular models of phonological rule systems. Computational Linguistics 20(3) (1994) 331-378

5. Heinz, J.: Computational phonology - part I: Foundations. Language and Linguistics Compass 5 (2011) 140-152

6. Gerdemann, D., Hulden, M.: Practical finite state optimality theory. In: Proceedings of the 10th International Workshop on Finite State Methods and Natural Language Processing. (2012) 10-19

7. Riggle, J.: Generation Recognition and Learning in Finite State Optimality Theory. PhD thesis, UCLA (2004)

8. Frank, R., Satta, G.: Optimality Theory and the generative complexity of constraint violability. Computational Linguistics 24(2) (1998) 307-315

9. Karttunen, L.: The proper treatment of optimality in computational phonology. In: Proceedings of the International Workshop on Finite State Methods in Natural Language Processing, Association for Computational Linguistics (1998) 1-12

10. Heinz, J.: Computational phonology - part II: Grammars, learning, and the future. Language and Linguistics Compass 5(4) (2011) 153-168

11. McCarthy, J.: Hidden Generalizations: Phonological Opacity in Optimality Theory. Equinox Publishing Ltd. (2007)

12. Baković, E.: Opacity and ordering. In Goldsmith, J.A., Riggle, J., Yu, A.C.L., eds.: The Handbook of Phonological Theory. second edn. Blackwell Publishers (2011)

13. McCarthy, J.: Sympathy and phonological opacity. Phonology 16 (1999) 331-399

14. Kiparsky, P.: Historical linguistics. In Dingwall, W.O., ed.: A Survey of Linguistic Science. University of Maryland Linguistics Program, College Park (1971)

15. Kiparsky, P.: Abstractness, opacity, and global rules. In Fujimura, O., Smith, D.L., eds.: Three Dimensions of Linguistic Theory. TEC (1973)

16. Kager, R.: Optimality Theory. Cambridge University Press (1999)

17. Graf, T.: Reference-set constraints as linear tree transductions via controlled optimality systems. In de Groote, P., Nederhof, M.J., eds.: Formal Grammar 2010/2011. Volume 7395 of Lecture Notes in Computer Science., Heidelberg, Springer (2012) 97-113

18. Bethin, C.Y.: Phonological rules in the nominative singular and genitive plural of the Slavic substantive declension. PhD thesis, University of Illinois, ChampaignUrbana (1978)

19. Kenstowicz, M., Kisseberth, C.: Generative Phonology: Description and Theory. Academic Press (1979)

20. Law, H.: Morphological structure of Isthmus Nahuat. International Journal of American Languages 24 (1958) 108-129

21. Hulstaert, G.: Grammaire du Lomongo. Musée royal de l'Afrique centrale (1961)

22. de Rijk, R.: Vowel interaction in Bizcayan Basque. Fontes Linguae Vasconum 2(5) (1970) 149-167 